\title{
ON THE PERMUTATIONAL REPRESENTATION OF GENERAL SETS OF OPERATIONS BY PARTITION LATTICES
}

BY

\author{
ALFRED L. FOSTER
}

Introduction. Let $U=\{\cdots, x, \cdots\}$ be a class (with or without structure), whose elements we may refer to as ground elements, and let $\Omega=\{\cdots, \omega, \cdots\}$ be a class of unary operations in-also called monotations of $-U$,

$$
x \rightarrow \omega(x)=\epsilon U \quad(\omega \in \Omega) .
$$

An $\Omega$ partition of $U$ is a logical partition of $U$ (into disjoint subsets or cells) in which the cells remain intact under all the (cellular) monotations induced in the class of cells by the various ground monotations $\omega \in \Omega$.

The familiar classical theory of imprimitivity sets is concerned with the study of the $\Omega$ partitions in the special case in which $\Omega$ is a group (or merely a set) of permutations $\left({ }^{1}\right)$; in this special case, for each given $\Omega$-partition the induced cellular monotations are (like the ground permutations $\omega$ ) themselves all necessarily cellular permutations.

The present communication shows that this latter theory may be cast in the considerably more general framework in which the set $\Omega$ may be a quite arbitrary set of monotations-not necessarily permutations. In this general case the various induced cellular monotations, corresponding to a given $\Omega$-partition, will no longer necessarily be permutations, as in the classical imprimitivity set case. In this present paper we shall, however, study the structure of just these permutational $\Omega$-partitions of $U$ for which all the induced cellular monotations are permutations $\left({ }^{2}\right)$. We shall also be concerned with the structure of the closely related more general class of univoque $\Omega$-partitions.

Each permutational $\Omega$-partition may be thought of as exhibiting the class $\Omega$ of monotations of $U$ as a set of permutations in the large of $U$. In this connection it is shown that under specified conditions (which are always satisfied if, for example, $U$ is finite) all $\Omega$-permutational partitions are "derivable" from a unique atomic partition, which is also $\Omega$-permutational; that is, any $\Omega$-permutational partition has this atomic partition as a "refinement." In the

Presented to the Society, October 30, 1948; received by the editors May 1, 1948.

(1) Here, as throughout the paper, the term "permutation" means simply a 1-1, and therefore reversible, transformation. In particular, $U$ need not be a finite set.

(2) Actually we consider the more general case of an operational algebra $(U, \Gamma)$ with a (possibly empty) class $\Gamma$ of operations. 
classical imprimitivity set case mentioned above this result is trivially true with 0 - that is, that partition whose cells are the individual ground elements of $U$-as atomic.

We have thus to do with various (homomorphic) representations of $\Omega$ by means of permutations, in which the product (=composition) of two $\omega$ 's of $\Omega$ is represented by the product of the corresponding cellular permutations. Appealing to the general notion of transforms of functions under coordinate changes, introduced in another communication $\left({ }^{3}\right)$, all invariants of the associated set of cellular permutations are seen to yield corresponding invariants of $\Omega$. In fact, the relationship of a general system of monotations to its various permutational representations is formally similar to the relationship of homomorphisms to isomorphisms in such algebraic disciplines as groups or rings.

The natural language of the subject is that of partition lattices, which are briefly reviewed at the outset. For completeness we include a derivation of the basic known Theorem 1, in terms of lemmas required for later use.

\section{Partition lattices}

1. Partitions. Let $U=\{\cdots, x, \cdots\}$ be a class of (ground) elements; we reserve small italic letters to denote elements of $U$, and italic capitals to denote subclasses of $U$.

A partition $A=\{\cdots, A, \cdots\}$ of $U$ is a class $A$ of pairwise disjoint non-empty subclasses $\cdots, A, \cdots$ of $U$ which cover $U$. These subclasses are called elements or cells of the partition $A$-written $A \in A$. For a partition $A$, then,

$$
\begin{array}{cc}
\sum A=U & \left(\sum=\text { class union }\right), \\
A A^{\prime}(=\text { intersection })=0 & \left(A \neq A^{\prime} ; A, A^{\prime} \in A\right) .
\end{array}
$$

As indicated, bold face $A, B$, and so on, are reserved for partitions.

We write $A=B$ (equality of partitions) only if $X \in A \rightleftarrows X \in B$, that is, if the partitions have the same cells. The notation

$$
x \equiv y(A)
$$

(read: $x$ congruent $y, \bmod A$ ) denotes that $x$ and $y$ lie in the same cell of the partition $\boldsymbol{A}$. More generally,

$$
X \equiv Y(A)
$$

denotes that the subsets $X$ and $Y$ are $\subseteq$ (class included in) the same cell of $A$.

If $\mathfrak{A}=\{\cdots, A, \cdots\}$ is a class of partitions, in addition to the obvious notation $A \in \mathfrak{A}$ we shall also write

(3) See [2]. All references in square brackets are listed in the bibliography. 


$$
A \in \in \mathfrak{A}
$$

(read: $A$ occurs in $\mathfrak{A}$ ) to mean: $A \in A$ for some $A \in \mathfrak{A}$. Further, if $F$ is a subset of $U$ and if $\mathfrak{A}$ is as above, we write

$$
A \in \in \mathfrak{A} \& F
$$

(read: the cell $A$ occurs in $\mathfrak{A}$ and in $F$ ) to mean

$$
A \in \in \mathfrak{A} \text { and } A F=A .
$$

The special partitions 0 (null partition) and 1 (universe partition) of $U$ are defined by

$$
\begin{aligned}
& x \equiv y(0) \\
& x \equiv y(1)
\end{aligned}
$$

only if $x=y$, for all $x, y$.

2. Lattices. The class $\mathfrak{U}=\{\cdots, \boldsymbol{X}, \cdots\}$ of all partitions of the class $U$ is converted into a partially ordered system $(\mathfrak{U}, \leqq)$ if one defines

$$
X \leqq Y
$$

(read: $\boldsymbol{X}$ is a refinement of $\boldsymbol{Y}$ ) to mean

$$
X Y=X \text { or } 0 \quad \text { for all } X \in X, Y \in Y .
$$

In fact, as is well known, one has the following theorem.

THEOREM A. The system $(\mathfrak{U}, \leqq)=(\mathfrak{u}, \cap, \cup)$ is a complete lattice.

By this is meant that any subset $\mathfrak{A} \subseteq \mathfrak{U}$ has both a greatest lower bound and a least upper bound, written

$$
\mathrm{glb}=\cap \mathfrak{A} \text { and } \mathrm{lub}=\cup \mathfrak{A},
$$

both $\in$ of $\mathfrak{u}$. Here $\cap$ and $\cup$ are defined in terms of $\leqq$ as in the case of general lattices (and conversely $\leqq$ is definable in terms of $\cap$ and $U$ ) (see [1]), namely: if $D=\cap \mathfrak{A}, M=\cup \mathfrak{A}$, then $D$ and $M$ have the definitive properties,

$$
D \leqq A \text { for all } A \in \mathfrak{A} \text {; and if } D^{\prime} \leqq A \text { for all } A \in \mathfrak{A} \text {, then } D^{\prime} \leqq D,
$$

and dually,

$A \leqq M$ for all $A \in \mathfrak{A}$; and if $A \leqq M^{\prime}$ for all $A \in \mathfrak{A}$, then $M \leqq M^{\prime}$.

The definitive properties (2.3) and (2.4) are not as convenient for our purpose as two lemmas which follow readily therefrom.

Lemma 1. Let $\mathfrak{A}=\left\{\cdots, A, A^{\prime}, \cdots\right\}$ be a class of partitions of $U$, $\mathfrak{U} \subseteq \mathfrak{U}$, and let $D=\cap \mathfrak{A}$. Each $D \in D$ is then a nonempty subset of $U$, and $D$ has one and only one "normal intersection" representation,

$$
D=\prod A=A A^{\prime} A^{\prime \prime} \cdots,
$$


that is, as the intersection of cells $A, A^{\prime}, A^{\prime \prime}, \cdots$, exactly one of which belongs to each partition $A(A \in \mathfrak{A})$. Conversely, if such a normal intersection of cells yields a nonempty subset of $U$, this subset is $\in D$.

While the lemma is fairly evident, because of its central role we shall prove it formally.

Proof. In all normal intersections let us agree to order the "factors" so that corresponding factors are cells of the same partition. Let

$$
C_{1}=A_{1} A_{1}^{\prime} A_{1}^{\prime \prime} \cdots, \quad C_{2}=A_{2} A_{2}^{\prime} A_{2}^{\prime} \cdots
$$

be any nonvoid normal intersection, where, for all $i, A_{1}^{(\mathfrak{l})}$ and $A_{2}^{(\mathfrak{l})}$ are then cells of the same partition $A \in \mathfrak{A}$. If $C_{1} \neq C_{2}$ then, for some $i, A_{1}^{(i)} \neq A_{2}^{(i)}$, and therefore $A_{1}^{(i)} A_{2}^{(i)}=0$, hence $C_{1} C_{2}=0$. Again if $x \in U$ and if $A_{x}^{(i)}$ is the cell of $A^{(i)}$ which contains $x$, then

$$
C_{x}=A_{x} A_{x}^{\prime} A_{x}^{\prime \prime} \ldots
$$

is a normal intersection containing $x$, and $C_{x}$ is nonvoid. We have thus shown that the totality of nonvoid normal intersections form a partition $\mathbf{C}$ of $U$, since the cells of $\boldsymbol{C}$ are pairwise disjoint and cover $U$.

There remains to show that $C=D$, that is, that

$$
A^{*} A A^{\prime} A^{\prime \prime} \ldots=A A^{\prime} A^{\prime \prime} \cdots \text { or } 0 \text {, }
$$

and that

$$
\text { if } D^{\prime} A^{*}=D^{\prime} \text { or } 0 \text { then } D^{\prime} A A^{\prime} A^{\prime \prime} \cdots=D^{\prime} \text { or } 0 \text {, }
$$

where, in (2.8) and (2.9), $A^{*}$ is any $\in \in \mathfrak{A}, D^{\prime}$ is any subset of $U$ and $A A^{\prime} A^{\prime \prime} \ldots$ is any $\in C$, that is, any nonempty normal intersection taken from $\mathfrak{A}$. The above (2.8) and (2.9) are simply the two parts of the definitive property (2.3) for $D$, after replacing $D$ by $C$. The truth of (2.8) is immediate since $A^{*}$ either is or is not identical with one of the factors $A^{(i)}$ : if it is so identical, the left of (2.8) obviously reduces to $A A^{\prime} A^{\prime \prime} \cdots$; if it is not it reduces to 0 since $A^{*} A^{(j)}=0$, where the component $A^{(j)}$ is determined by $A^{*} \in A^{(j)} \in \mathfrak{X}$. Similarly (2.9) is immediate since its premise insures that $D^{\prime} A^{(i)}=D^{\prime}$ or 0 for each factor $A^{(j)}$; if $D^{\prime} A^{(j)}=D^{\prime}$ for all $j$, the desired conclusion follows with the result $D^{\prime}$, and otherwise it follows with the result 0 . This completes the proof of Lemma 1.

We state a second useful quite evident consequence of the definitive property (2.4) of lub without proof:

LEMma 2. In the notation of Lemma 1 and of (1.5), let $M=\cup \mathfrak{A}$, let $M \in M$, and let $\mathscr{X}=\{\cdots, X, \cdots\}$ be the class of all cells occurring in $\mathfrak{A}$ and in $M$ (that is, $X \in \in \mathfrak{A}$, and $X \subseteq M$ ). Then $\mathcal{X}$ cannot be expressed as a union of subsets

$$
\mathscr{X}=\mathscr{X}_{1}+\mathscr{X}_{2}
$$


neither of which is empty and which are "disjoint," that is, which satisfy

$$
X_{1} X_{2}=0 \quad \text { for all } X_{1} \in \mathcal{X}_{1}, X_{2} \in \mathcal{X}_{2} \text {. }
$$

Sublattices. For later reference we make a few observations concerning sublattices. The intersection of a set of sublattices-respectively of a set of complete sublattices-of $(\mathfrak{U}, \cap, \cup)$ is again a sublattice-respectively a complete sublattice. If $\mathfrak{A}$ is a subset of $\mathfrak{U}, \mathfrak{A} \subseteq \mathfrak{U}$, the simple-respectively the complete-sublattice generated by $\mathfrak{A}$ is denoted by $|\mathfrak{A}|=(|\mathfrak{A}|, \cap, \cup)$ respectively by $\|\mathfrak{A}\|$. Here $|\mathfrak{A}|$-respectively $\| \mathfrak{A}||$-is the intersection of allrespectively of all complete-sublattices of $\mathfrak{U}$ which contain the set $\mathfrak{A}$. $|\mathfrak{A}|$ and $\|\mathfrak{A}\|$ are called the simple and complete lattice closures of $\mathfrak{A}$. One has

$$
\mathfrak{A} \subseteq|\mathfrak{A}| \subseteq\|\mathfrak{X}\| \subseteq \mathfrak{u} .
$$

It is readily seen that the glb and also the lub of either type of closure is the same as that of $\mathfrak{A}$,

$$
\begin{aligned}
& \cap \mathfrak{A}=\cap|\mathfrak{A}|=\cap\|\mathfrak{A}\|, \\
& \cup \mathfrak{A}=\cup|\mathfrak{A}|=\cup\|\mathfrak{A}\| .
\end{aligned}
$$

One should however observe that, while in the complete case $\cap\|\mathfrak{A}\|$ and $\cup\|\mathfrak{A}\|$ are necessarily elements of the set $\|\mathfrak{A}\|$, the same will not in general be true for the simple closure $|\mathfrak{A}|$.

\section{Partitions of AN ALGEBRA}

3. Fundamental notions. By a (general operational) algebra $(U, \Gamma)$ we understand a class $U=\{\cdots, x, \cdots\}$ together with a set $\Gamma$ $=\{\cdots, \gamma, \cdots\}$ of operations (=functions) $\gamma=\gamma_{i}$ whose range is $U$,

$$
\gamma_{i}=\gamma_{i}\left(x_{1}, x_{2}, \cdots, x_{n_{i}}\right)=\epsilon U
$$

for all $x_{1}, x_{2}, \cdots \in U$. We permit $\Gamma$ to be empty, and hence a pure class is a special case of an algebra. The argument numbers $n_{i}$ of the $\gamma_{i}$ need not necessarily be finite.

As previously noted, a $\gamma=\gamma(x)$ of a single argument is called a monotation (of $U$ ). A monotation $\gamma$ is univoque-also called schlicht-if

$$
x \neq y \rightarrow \gamma(x) \neq \gamma(y) \text {. }
$$

A permutation is a schlicht monotation in which $\gamma(x)=y$ has a solution $y \in U$ for each $x \in U$. ( $U$ need not be finite.) The product $\gamma \gamma^{\prime}$ of monotations is the composite monotation $\gamma\left(\gamma^{\prime}(x)\right)$.

By a partition ( $\Gamma$-partition) $A$ of the algebra $(U, \Gamma)$ we mean a partition $A$ of $U$ such that

$$
x_{1} \equiv y_{1}, x_{2} \equiv y_{2}, \cdots,(A) \rightarrow \gamma\left(x_{1}, x_{2}, \cdots\right) \equiv \gamma\left(y_{1}, y_{2}, \cdots\right)(A)
$$

for all $\gamma \in \Gamma$. 
If $\boldsymbol{A}$ is a partition of $(U, \Gamma)$, each $\gamma \in \Gamma$ induces an operation $\gamma$ in $\boldsymbol{A}$, defined by:

(3.4) $\gamma\left(X_{1}, X_{2}, \cdots\right)=Y$ if $\gamma\left(x_{1}, x_{2}, \cdots\right)=y\left(x_{i} \in X_{i} ; X_{i}\right.$ and $\left.Y \in A\right)$.

It follows from the definition of $\Gamma$-partition that $Y$ in (3.4) depends only on the $X_{i}$, and not on the choice of the $x_{i} \in X_{i}$.

The algebra whose elements are the cells comprising $A$ and whose class $\boldsymbol{\Gamma}$ of operations consists of the set of all induced $\gamma$, we call the cell algebra $(\boldsymbol{A}, \boldsymbol{\Gamma})$, also written $(U, \Gamma) / \boldsymbol{A}$. The mapping

$$
\begin{aligned}
& x \rightarrow X \\
& \gamma \rightarrow \gamma
\end{aligned} \quad(x \in X, X \in A)
$$

is then a homomorphic map

$$
(U, \Gamma) \rightarrow(A, \Gamma)
$$

of the algebra $(U, \Gamma)$ onto the cell algebra, that is,

$$
\begin{aligned}
\gamma\left(x_{1}, x_{2}, \cdots\right)=y \rightarrow \gamma\left(X_{1}, X_{2}, \cdots\right) & =Y, \\
& \left(x_{i} \in X_{i}, y \in Y ; X_{i}, Y \in A\right) .
\end{aligned}
$$

Furthermore for any monotation $\omega_{1}=\omega_{1}(x), \omega_{2}=\omega_{2}(x)$ which $\Gamma$ may contain, it is evident that the homomorphism established by the mapping (3.5) preserves composition, that is, insures that

$$
\omega_{1} \omega_{2} \rightarrow \omega_{1} \omega_{2} \text {. }
$$

It is often desirable to regard $\Gamma$ as the union

$$
\Gamma=\Psi+\Omega
$$

of two subclasses $\Psi=\{\cdots, \psi, \cdots\}$ and $\Omega=\{\cdots, \omega, \cdots\}$, corresponding possibly to some fundamental distinction between the $\psi \in \Psi$ and the $\omega \in \Omega$. In this case we also write

$$
(U, \Gamma)=(U, \Psi: \Omega)
$$

For given $\Omega \subseteq \Gamma$, by

$$
(\boldsymbol{A}, \mathbf{\Gamma}) q u a \mathbf{\Omega}
$$

we mean the cell algebra $(A, \Gamma)$ conceived only with respect to the operations $\Omega$.

4. Lattice of algebra partitions. Let $(U, \Gamma)$ be an algebra, and let $\mathfrak{u}_{\Gamma}=\{\cdots, A, \cdots\}$ denote the totality of partitions ( $\Gamma$-partitions) of $(U, \Gamma)$. Corresponding to Theorem A one has:

Theorem 1. $\left(\mathfrak{U}_{\Gamma}, \cap, \cup\right)$ is a (complete) lattice (= sublattice of $(\mathfrak{U}, \cap, \cup)$ ).

Proof. Let $\mathfrak{A}_{\Gamma} \subseteq \mathfrak{U}_{\Gamma}, D=\cap \mathfrak{A}_{\Gamma}, \boldsymbol{M}=\cup \mathfrak{A}_{\Gamma}$. Both $D$ and $\boldsymbol{M}$ are $\in \mathfrak{U}$ (Theorem 
A); we must show that they are furthermore $\in \mathfrak{U}_{\Gamma}$.

Let us first take $D$. Here one must show that

$$
x_{1} \equiv y_{1}, x_{2} \equiv y_{2}, \cdots(D) \rightarrow \gamma\left(x_{1}, x_{2}, \cdots\right) \equiv \gamma\left(y_{1}, y_{2}, \cdots\right)
$$

(for all $\gamma \in \Gamma$ ).

This is immediate since the premise of (4.1) implies that $x_{1} \equiv y_{1}, x_{2} \equiv y_{2}, \ldots$ $(A)$ for each $A \in \mathfrak{A}_{\Gamma}$; this in turn implies that

$$
\gamma\left(x_{1}, x_{2}, \cdots\right) \equiv \gamma\left(y_{1}, y_{2}, \cdots\right)(A),
$$

$\left(A \in \mathfrak{A}_{\Gamma} ; \gamma \in \Gamma\right)$,

since each $A \in \mathfrak{A}_{\Gamma}$ is by hypothesis a partition of $(U, \Gamma)$. The conclusion of (4.1) follows at once from (4.2).

Next consider $M$. We must show that

$$
x_{1} \equiv y_{1}, x_{2} \equiv y_{2}, \cdots(\boldsymbol{M}) \rightarrow \gamma\left(x_{1}, x_{2}, \cdots\right) \equiv \gamma\left(y_{1}, y_{2}, \cdots\right)(\boldsymbol{M}) \text {, }
$$

(for all $\gamma \in \Gamma$ ).

We first prove the following lemma.

LemMa 3. For each $\gamma \in \Gamma$ which is a monotation, $\gamma=\gamma(x)$,

$$
x \equiv y(\boldsymbol{M}) \rightarrow \gamma(x) \equiv \gamma(y)(\boldsymbol{M}) .
$$

Proof. Let $x$ and $y$ be $\in M(M \in M)$. We must show that $\gamma(x) \equiv \gamma(y)(M)$. Suppose this false, and let $\gamma(x) \in M_{1}\left(M_{1} \in M\right)$; then $\gamma(y) \notin M_{1}$. Separate all cells occurring in both $\mathfrak{A}_{\Gamma}$ and $M$ (Lemma 2 ) into classes $\mathcal{X}^{\prime}$ and $\mathcal{X}^{\prime \prime}$ where

$$
\begin{array}{lll}
A \in \mathcal{X}^{\prime} & \text { if } & \gamma(A) M_{1}=\gamma(A), \\
A \in \mathcal{X}^{\prime \prime} & \text { if } & \left.\gamma(A) \nsubseteq M_{1} \text { (equivalent to } \gamma\left(A_{1}\right) M_{1}=0\right) .
\end{array}
$$

By our hypothesis on $\gamma(x)$ and $\gamma(y)$, neither $\mathcal{X}^{\prime}$ nor $\mathcal{X}^{\prime \prime}$ is empty. Moreover

$$
A^{\prime} A^{\prime \prime}=0 \quad \text { (for all } A^{\prime} \in \mathcal{X}^{\prime}, A^{\prime \prime} \in \mathcal{X}^{\prime \prime} \text { ), }
$$

for, if not, for any $z \in A^{\prime} A^{\prime \prime}, \gamma(z)$ would have to be both $\in M_{1}$ and $€ M_{1}$, by (4.5) and the definition of $\Gamma$ partition. We thus have a contradiction with Lemma 2 , and Lemma 3 is proved

We return to the proof of (4.3). Let $\gamma$ be any member of $\Gamma$, and let $a_{1}, b_{1}, a_{2}, b_{2}, \cdots$ be given elements of $U$ such that $a_{1} \equiv b_{1}, a_{2} \equiv b_{2}, \cdots(\boldsymbol{M})$. We must show that

$$
\gamma\left(a_{1}, a_{2}, \cdots\right) \equiv \gamma\left(b_{1}, b_{2}, \cdots\right)(M) .
$$

Set

$$
\gamma^{\prime}(x)=\text { def. }=\gamma\left(x, a_{2}, a_{3}, \cdots\right)
$$

and let $\Gamma^{\prime}=\Gamma+\gamma^{\prime}$ (adjunction of $\gamma^{\prime}$ to $\Gamma$ ). It is evident that each $\Gamma$-partition of $U$ is also a $\Gamma^{\prime}$ partition, and hence by (4.4) and Lemma 3 we have 
that is,

$$
\boldsymbol{\gamma}^{\prime}\left(a_{1}\right) \equiv \boldsymbol{\gamma}^{\prime}\left(b_{1}\right)(\boldsymbol{M})
$$

$$
\gamma\left(a_{1}, a_{2}, a_{3}, \cdots\right) \equiv \gamma\left(b_{1}, a_{2}, a_{3}, \cdots\right)(M) .
$$

Setting $\gamma^{\prime \prime}(x)=\gamma\left(b_{1}, x, a_{3}, \cdots\right)$ and repeating the preceding argument we get

$$
\gamma\left(b_{1}, a_{2}, a_{3}, \cdots\right) \equiv \gamma\left(b_{1}, b_{2}, a_{3}, \cdots\right)(M) .
$$

The desired result (4.3) is then obtained by inductive repetition of this procedure, which completes the proof of Theorem 1.

We observe that in all cases the lattice $\left(\mathfrak{U}_{r}, \cap, \cup\right)$ has the same least and greatest elements as $(\mathfrak{u}, \cap, \cup)$, namely 0 and 1 .

5. The glb under monotations. In this section we assume that the algebra $(U, \Gamma)$ contains at least one $\omega \in \Gamma$ which is a monotation, $\omega=\omega(x)$, and suppose $\Omega=\{\cdots, \omega, \cdots\}$ to be any set of monotations $\Omega \subseteq \Gamma$. We may write

$$
\begin{aligned}
\Gamma & =\Psi+\Omega & \text { (union; also written } \Psi: \Omega \text { ), } \\
\Psi \Omega & =0 & \text { (intersection). }
\end{aligned}
$$

(Here any $\psi \in \Psi$ may or may not be a monotation. Again $\Psi$ may, as a special case, be empty.)

Related to Lemma 1 we have the following theorem.

TheOREM 2. Let: $\mathfrak{A}_{\Gamma}=\left\{\cdots, A^{(i)}, \cdots\right\}$ be any set of $\Gamma$-partitions of the algebra $(U, \Gamma)=(U, \Psi: \Omega), \mathfrak{A}_{\Gamma} \subseteq \mathfrak{U}_{\Gamma} ; D=\cap \mathfrak{A}_{\Gamma}, D \in D, \omega \in \Omega$ and

$$
D=\prod A^{(i)}
$$

be the normal intersection representation of $D$. Then in the cell algebra $(D, \Gamma)=$ $(D, \Psi: \Omega)$,

$$
\omega(D)=\omega\left(\prod A^{(i)}\right)=\prod \omega\left(A^{(i)}\right) .
$$

Proof. We have $\omega(D)=D^{\prime}=\in D$ (Theorem 1). Now $D$ is nonvoid, and

$$
x \in D\left\{\begin{array}{l}
\rightarrow \omega(x) \in D^{\prime}, \\
\rightarrow x \in A^{(i)} \text { for all } i, \rightarrow \omega(x) \in \omega\left(A^{(i)}\right) \text { for all } i, \\
\rightarrow \omega(x) \in \prod \omega\left(A^{(i)}\right) .
\end{array}\right.
$$

From (5.3), $\Pi \omega\left(A^{(i)}\right)$ is nonvoid, and is seen to be the normal intersection representation of some element of $D$ (Lemma 1 ). This element can however only be $D^{\prime}$, since $D^{\prime}$ and $\amalg \omega\left(A^{(i)}\right)$ are not disjoint, by (5.3). This completes the proof of Theorem 2.

For convenient reference, using the notation of (1.5) we state the following incidence properties, which are immediate consequences of the definition of $\Gamma$-partition: 
Lemma 4. Let $A, B, C$, and so on, be any class of $\in \in \mathfrak{U}_{\Psi: \Omega}$, and let $\omega \in \Omega$. Then

$$
\begin{aligned}
& x \in A B C \cdots \rightarrow \omega(x) \in \omega(A) \omega(B) \omega(C) \cdots, \\
& A B C \cdots \neq 0 \rightarrow \omega(A) \omega(B) \omega(C) \cdots \neq 0 .
\end{aligned}
$$

\section{SCHLICHT AND PERMUTATIONAL PARTITIONS}

6. The glb. Using the notation of $\$ 5$, we now define a partititon $A$ of $(U, \Gamma)=(U, \Psi: \Omega)$ to be $\Omega$-univoque, also called $\Omega$-schlicht if the cell algebra $(A, \Gamma) q u a \Omega$ is schlicht (see (3.2)); that is, if

$$
X \neq Y \rightarrow \omega(X) \neq \omega(Y),
$$$$
(X, Y \in A ; \omega \in \Omega) .
$$

If in addition to (6.1), for $\omega \in \mathbf{\Omega}$ and for each $A \in A$

$$
\omega(X)=A
$$

has a solution $X \in A$, we call $A$ an $\Omega$-permutational partition of the algebra $(U, \Psi: \Omega)$.

In both $\Omega$-schlicht and $\Omega$-permutational partitions the glb and lub play nonsymmetrical roles. In this section we consider only the glb, and in $\$ 7$ the lub.

Let $\mathfrak{S}_{\Psi: \Omega}, \mathfrak{B}_{\Psi: \Omega}$ denote the class of all $\Omega$-schlicht and the class of all $\Omega$-permutational partitions of $(U, \Psi: \Omega)$ respectively. Where no danger of ambiguity exists we shall also abbreviate these to $\mathfrak{S}_{\Omega}$ and $\mathfrak{B}_{\Omega}$. Of course

$$
\mathfrak{P}_{\Omega} \subseteq \mathfrak{S}_{\Omega} \subseteq \mathfrak{u}_{\boldsymbol{\Gamma}} \subseteq \mathfrak{u}
$$

It is evident that in all cases

$$
1 \in \mathfrak{B}_{\Omega}, \quad 1 \in \mathfrak{S}_{\Omega} .
$$

However 0 is only then $\in \Im_{\Omega}$, respectively $\in \mathfrak{P}_{\Omega}$, if each $\omega \in \Omega$ is schlicht, respectively a permutation (of $U$ ).

As an important special case it is furthermore clear that, in case $U$ is a finite class, the notions schlicht and permutational coincide, and in particular

$$
\mathfrak{S}_{\Omega}=\mathfrak{P}_{\Omega} \text {. }
$$

TheOREM 3. The glb of a class $\mathfrak{A}_{\Omega}$ of $\Omega$-schlicht partitions of $(U, \Psi: \Omega)$ is also an $\Omega$-schlicht partition,

$$
\mathfrak{A}_{\Omega} \subseteq \mathfrak{S}_{\Omega} \rightarrow \cap \mathfrak{A}_{\Omega} \in \mathfrak{S}_{\Omega} .
$$

Proof. Let $D=\cap \mathfrak{A}_{\Omega}\left(=\mathfrak{A}_{\Psi: \Omega}\right)$. We must show for each $\omega \in \Omega$ and for all $D_{1}, D_{2} \in D$ that

$$
D_{1} \neq D_{2} \rightarrow \omega\left(D_{1}\right) \neq \omega\left(D_{2}\right)
$$

Let

$$
D_{1}=A A^{\prime} A^{\prime \prime} \ldots=\prod A^{(i)}, \quad D_{2}=B B^{\prime} B^{\prime \prime} \ldots=\prod B^{(i)}
$$


be the normal intersection representation of $D_{1}, D_{2}$ respectively (Lemma 1 ), where we assume the "factors" in each case arranged so that for each $i$, corresponding factors $A^{(i)}, B^{(i)}$ belong to the same partition $A^{(i)} \in \mathfrak{A}_{\Omega}$. Then since $D_{1} \neq D_{2}$, at least one of the factors of $D_{1}$ is different from the corresponding factor of $D_{2}$. We may assume the factors so ordered that $A \neq B$, that is,

$$
A B=0 \text {. }
$$

Now

$$
\begin{aligned}
& \omega\left(D_{1}\right)=\omega(A) \omega\left(A^{\prime}\right) \omega\left(A^{\prime \prime}\right) \cdots=\in D, \\
& \omega\left(D_{2}\right)=\omega(B) \omega\left(B^{\prime}\right) \omega\left(B^{\prime \prime}\right) \cdots=\in D,
\end{aligned}
$$

by Theorems 2 and 1 . But since $A$ and $B$ are cells of the same $A \in \mathfrak{A}_{\Omega}$, and since by hypothesis each $A \in \mathfrak{A}_{\Omega}$ is $\Omega$-schlicht, we have that $\omega(A) \omega(B)=0$. Hence $\omega\left(D_{1}\right) \omega\left(D_{2}\right)=0$, by (6.8); that is, $\omega\left(D_{1}\right)$ and $\omega\left(D_{2}\right)$ are different elements of $D$, which proves Theorem 3 .

The lub companion proposition to Theorem 3 is unfortunately not generally true. In fact, as the following example shows, the lub $A \cup B$ need not be $\Omega$-schlicht, even though $A$ and $B$ are.

Example. $U=\left\{1 ; 2,2^{\prime}, 2^{\prime \prime}, \ldots ; 3,3^{\prime}, 3^{\prime \prime}, \ldots ; 4,4^{\prime}, 4^{\prime \prime}, \ldots\right.$; $\left.5,5^{\prime}, 5^{\prime \prime}, \cdots\right), \Psi$ empty, $\Omega=\omega$, where

$$
\omega(1)=\omega(2)=2^{\prime} ; \omega(k)=k^{\prime}, \omega\left(k^{\prime}\right)=k^{\prime \prime}, \omega\left(k^{\prime \prime}\right)=k^{\prime \prime \prime}, \cdots
$$

(for $k=2,3,4,5)$.

The following two are seen to be $\omega$-schlicht partitions of $U$ :

$$
\begin{array}{r}
A=\{1,2\},\{3\},\left\{3^{\prime}, 4\right\},\left\{5,2^{\prime}\right\},\left\{3^{\prime \prime}, 4^{\prime}\right\},\left\{5^{\prime}, 2^{\prime \prime}\right\},\left\{3^{\prime \prime \prime}, 4^{\prime \prime}\right\},\left\{5^{\prime \prime}, 2^{\prime \prime \prime}\right\}, \cdots \\
B=\{1,2\},\{3\},\left\{3^{\prime}\right\},\{4,5\},\left\{2^{\prime}\right\},\left\{3^{\prime \prime}\right\},\left\{4^{\prime}, 5^{\prime},\right\},\left\{2^{\prime \prime}\right\}, \\
\quad\left\{3^{\prime \prime \prime}\right\},\left\{4^{\prime \prime}, 5^{\prime \prime}\right\},\left\{2^{\prime \prime \prime}\right\}, \cdots .
\end{array}
$$

Here $M=A \cup B$ is the partition

$$
M=\{1,2\},\{3\},\left\{3^{\prime}, 4,5,2^{\prime}\right\},\left\{3^{\prime \prime}, 4^{\prime}, 5^{\prime}, 2^{\prime \prime}\right\}, \cdots
$$

which is not $\omega$-schlicht, since

$$
2 \not \equiv 3(\boldsymbol{M}) \text { but } \omega(2) \equiv \omega(3)(\boldsymbol{M}) .
$$

In view of this circumstance one may conveniently restate Theorem 3 in the stronger form:

THEOREM $3^{\prime}$. The set $\mathfrak{S}_{\Omega}=\mathfrak{S}_{\Psi: \Omega}$ of all $\Omega$-schlicht partitions of an algebra $(U, \Psi: \Omega)$ form a complete $(\mathrm{glb})$ semi-lattice $\left(\Im_{\Omega}, \cap\right)$.

For $\Omega$-schlicht partitions, Lemma 4 has the following two immediate lemmas as a converse. 
Lemma 5. Let $A, B, C$, and so on, be any class of $\in \in S_{\Omega}$, and let $\omega \in \Omega$. Then

$$
x \notin A B C \cdots \rightarrow \omega(x) \notin \omega(A) \omega(B) \omega(C) \cdots .
$$

Proof. From the premise of (6.9), for some member $A$ of the given class, $x \notin A$. If now $A^{\prime}$ is defined by: $x \in A^{\prime}$, where $A$ and $A^{\prime}$ are cells of the same partition $A \in \mathcal{S}_{\Omega}$, we have

$$
A A^{\prime}=0, \quad \omega(x) \in \omega\left(A^{\prime}\right), \quad \omega(A) \omega\left(A^{\prime}\right)=0,
$$

since $A$ is $\Omega$-schlicht. Hence $\omega(x) € \omega(A)$, and the lemma is proved.

Lemma 6. Let $A, B, C$, and so on, be any class of cells which are $\in \in \mathfrak{S}_{\Omega}$, let $\omega \in \Omega$ and let $E=E_{\omega}$ be the intersection

$$
E=E_{\omega}=\omega(A) \omega(B) \omega(C) \cdots .
$$

Then

$$
A B C \cdots=0 \rightarrow E=0
$$

or else E consists solely of non-target points under $\omega$.

Here $y \in U$ is a non-target point under $\omega$ if no $x \in U$ exists such that $\omega(x)=y$.

Proof. Suppose $E \neq 0$, and $z \in E$. If $z$ were a target point under $\omega$, let $x$ be such that $\omega(x)=z$. Then $z \in \omega(A), z \in \omega(B)$, and so on, and hence $x \in A$ (otherwise Lemma 5 would be contradicted), $x \in B$, and so on. That is, $x \in A B C \cdots$, which contradicts our premise that $A B C \cdots=0$. This proves Lemma 6.

Let $\mathfrak{A}_{\Omega}$ be a class of $\Omega$-schlicht partitions, $\mathfrak{A}_{\Omega} \subseteq \mathfrak{S}_{\Omega}$. We say that $\mathfrak{A}_{\Omega}$ satisfies the target condition if for each $\omega \in \Omega$,

$$
\prod A^{(i)}=0 \rightarrow \prod \omega\left(A^{(i)}\right)=0
$$

for each (empty) normal intersection, that is, where the cells $A^{(1)}, A^{(2)}, \ldots$ are chosen, exactly one from each partition $A \in \mathfrak{A}_{\Omega}$.

While the glb of any set of $\Omega$-schlicht partitions is again $\Omega$-schlicht (Theorem 3 or $3^{\prime}$ ), the corresponding proposition for $\Omega$-permutational partitions is generally not true-not even for a finite set of partitions-as may be seen from the following example.

EXAMPLE. Let $U=\left\{0, \pm 1, \pm 2, \cdots ; \alpha_{1}, \alpha_{2}, \alpha_{3}, \cdots\right\}, \Psi$ empty, $\Omega=\omega$, where

$$
\begin{aligned}
\omega(x) & =x+1 & \text { for any whole number } x, \\
\omega\left(\alpha_{k}\right) & =\alpha_{k+1} & (k=1,2, \cdots) .
\end{aligned}
$$

(Here $(U, \omega)$ is not a permutation since $\alpha_{1}$ is not a target element; $(U, \omega)$ is however schlicht.) It is seen that 


$$
A=\cdots, A_{-2}, A_{-1}, A_{0}, A_{1}, A_{2}, \cdots
$$

and

$$
B=\cdots, B_{-2}, B_{-1}, B_{0}, B_{1}, B_{2}, \cdots,
$$

where the cells $A_{n}$ of $A$ and $B_{n}$ of $B$ are defined by

$$
\begin{array}{ll}
A_{n}=B_{n}=\{n\}, & \text { for } n \leqq 0, \\
A_{n}=\left\{n, \alpha_{n}\right\}, & \text { for } n \geqq 1, \\
B_{1}=\{1\}, \quad B_{n+1}=\left\{n+1, \alpha_{n}\right\}, & \text { for } n \geqq 1,
\end{array}
$$

are two $\omega$-permutational partitions of $U$. However their glb is not $\omega$-permutational, since

$$
A \cap B=0
$$

and $\mathbf{0}$, as observed above, is not an $\omega$-permutational partition of $U$. (Note: In this example the lub $A \cup B$ is also not an $\omega$-permutational partition-in fact it is not even $\omega$-schlicht!)

In this example we further note that the set $A, B$ does not satisfy the target condition, since

$$
A_{0} B_{1}=0 \text { but } \omega\left(A_{0}\right) \omega\left(B_{1}\right) \neq 0 .
$$

This illustrates the following theorem.

THEOREM 4. If $\mathfrak{A}_{\Omega}$ is a class of $\Omega$-permutational partitions, $\mathfrak{A}_{\Omega} \subseteq \mathfrak{B}_{\Omega}, a$ necessary and sufficient condition for the glb of $\mathfrak{A}_{\Omega}$ to be $\Omega$-permutational, $\cap \mathfrak{A}_{\Omega} \in \mathfrak{P}_{\Omega}$, is that $\mathfrak{A}_{\Omega}$ satisfy the target condition.

Proof. Necessity. If $\mathfrak{A}_{\Omega} \subseteq \mathfrak{P}_{\Omega}$ but the target condition is not satisfied, then for some (empty) normal intersection and for some $\omega \in \Omega$,

$$
A^{(1)} A^{(2)} \cdots=0 \text { but } \omega\left(A^{(1)}\right) \omega\left(A^{(2)}\right) \cdots \neq 0 .
$$

Then, by Theorem 2,

$$
\boldsymbol{\omega}\left(A^{(1)}\right) \omega\left(A^{(2)}\right) \cdots=D=\in D \quad\left(D=\cap \mathfrak{A}_{\Omega}\right) .
$$

By Lemmas 1 and 6, $D$ is nonvoid and consists exclusively of nontarget points under $\omega$. Hence there exists no $D^{\prime} \in D$ such that $\omega\left(D^{\prime}\right)=D$; that is, $D$ is not $\Omega$-permutational. This proves the necessity of the target condition.

Sufficiency. Suppose $\mathfrak{A}_{\Omega} \subseteq \mathfrak{P}_{\Omega}$ and the target condition is satisfied. Now $D=\cap \Re_{\Omega}$ is $\Omega$-schlicht, $D \in \widetilde{S}_{\Omega}$, by Theorem 3 , and hence we have only to show that for each $D \in D$ and for each $\omega \in \Omega$ there exists a $D^{\prime} \in D$ such that

$$
\omega\left(D^{\prime}\right)=D \text {. }
$$

For given $D$, let

$$
D=A^{(1)} A^{(2)} \cdots
$$


be the normal intersection representation of $D$ (Lemma 1). Since by hypothesis each $A \in \mathfrak{A}_{\Omega}$ is $\Omega$-permutational, there exists for each index $i$ a cell $B^{(i)}$ belonging to the same $A \in \mathfrak{A}_{\Omega}$ as $A^{(i)}$, and such that

$$
\omega\left(B^{(i)}\right)=A^{(i)} \text {, }
$$

and hence

$$
\prod \omega\left(B^{(i)}\right)=\prod A^{(i)}=D .
$$

Since $D$ is nonvoid,

$$
B^{(1)} B^{(2)} B^{(3)} \cdots=D^{\prime}
$$

is also nonvoid, for otherwise the target condition would not be satisfied. Furthermore $D^{\prime}$ is $\in D$ (Lemma 1 ), and moreover

$$
\omega\left(D^{\prime}\right)=\omega\left(B^{(1)}\right) \omega\left(B^{(2)}\right) \cdots=D,
$$

by Theorem 2. This shows that (6.15) has a solution $D^{\prime}$ and completes the proof of Theorem 4.

TheOREM 5. Let $\mathfrak{A}_{\Omega}^{\prime} \subseteq \mathfrak{A}_{\Omega} \subseteq \mathfrak{S}_{\Omega}$. If $D=\cap \mathfrak{A}_{\Omega}$ is $\in \mathfrak{B}_{\Omega}$, then $D^{\prime}=\cap \mathfrak{A}_{\Omega}^{\prime}$ is also $\in \mathfrak{B} \Omega$.

Proof. $D^{\prime}$ is $\in S_{\Omega}$ (Theorem 3). Again $D \leqq D^{\prime}$, by definition of glb. Hence for $D^{\prime} \in D^{\prime}$ there exists a $D \in D$ such that $D \subseteq D^{\prime}$. If $\omega\left(D_{1}\right)=D$ and if $D_{1}^{\prime}$ is an $\in D^{\prime}$ such that $D_{1} \subseteq D_{1}^{\prime}$, then clearly $\omega\left(D_{1}^{\prime}\right)=D^{\prime}$, since

$$
x \in D_{1} \rightarrow \omega(x) \in \omega\left(D_{1}\right)=D,
$$

and thus $\omega(x) \in D^{\prime}$. Hence $D^{\prime}$ is $\Omega$-permutational, and the theorem is proved.

Taking $\mathfrak{U}_{\Omega}^{\prime}$ as $A \in \mathfrak{U}_{\Omega}$, Theorem 5 has the immediate corollary.

Corollary 1. A necessary condition for a class $\mathfrak{A}_{\Omega}$ of $\Omega$-schlicht partitions to have an $\Omega$-permutational glb is that each partition comprising $\mathfrak{A}_{\Omega}$ be $\Omega$-permutational. In symbols,

$$
\text { if } \mathfrak{A}_{\Omega} \subseteq \mathfrak{S}_{\Omega} \text { and if } D=\cap \mathfrak{A}_{\Omega} \text { is } \in \mathfrak{B}_{\Omega} \text {, then } \mathfrak{A}_{\Omega} \subseteq \mathfrak{P}_{\Omega} \text {. }
$$

Again, from Theorems 4 and 5 we have:

Corollary 2. If $\mathfrak{A}_{\Omega}^{\prime} \subseteq \mathfrak{A}_{\Omega} \subseteq \mathfrak{B}_{\Omega}$ and if $D=\cap \mathfrak{A}_{\Omega}$ is $\in \mathfrak{B}_{\Omega}$, then $\mathfrak{U}_{\Omega}^{\prime}$ satisfies the target condition and $D^{\prime}=\cap \mathfrak{A}_{\Omega}^{\prime}$ is $\in \mathfrak{B}_{\Omega}$.

7. The lub. We turn now to the lub of sets of $\Omega$-permutational partitions. As we have seen (see $\S 6$, following Theorem 3 ), in the case of a class $\mathfrak{U}_{\Omega}$ of $\Omega$-schlicht partitions, the glb of $\mathfrak{A}_{\Omega}$ is always $\Omega$-schlicht, while the lub need not be. In the stronger case of a class $\mathfrak{A}_{\Omega}$ of $\Omega$-permutational partitions, the situation is considerably different. Here, as we have already seen, not even $\cap \mathfrak{A}_{\Omega}$ need be $\Omega$-permutational. However in this case we shall show: if the $\mathrm{glb}$ is $\Omega$-permutational, so also is the lub. 
TheOREM 6. Let $\mathfrak{A}_{\Omega}$ be a set of $\Omega$-permutational partitions. If glb $\mathfrak{A}_{\Omega}$ is $\Omega$ permutational (or, equivalently, if $\mathfrak{A}_{\Omega}$ satisfies the target condition) the lub is also $\Omega$-permutational:

$$
\mathfrak{A}_{\Omega} \subseteq \mathfrak{B}_{\Omega} \text { and } \cap \mathfrak{A}_{\Omega}=\in \mathfrak{B}_{\Omega} \rightarrow \cup \mathfrak{A}_{\Omega}=\in \mathfrak{B}_{\Omega} .
$$

Proof. Let $M=\cup \mathfrak{A}_{\Omega}\left(=\cup \mathfrak{A}_{\Psi: \Omega}\right)$. Now $M \in \mathfrak{U}_{\Gamma}\left(=\mathfrak{U}_{\Psi: \Omega}\right)$, by Theorem 1; we must show that $M$ is also $\in \mathfrak{B}_{\Omega}$. We shall first show that

$$
M \in \mathfrak{S}_{\Omega} \text {. }
$$

Let $M_{0}$ be $\in M, \omega$ be $\in \Omega$, and let $M_{0}^{\prime}$ be defined (Theorem 1 ) by

$$
\omega\left(M_{0}\right)=M_{0}^{\prime}
$$

Let

$$
M_{0}, M_{1}, M_{2}, \cdots
$$

be the class of all distinct $\in M$ satisfying (7.2), that is, such that

$$
\omega\left(M_{0}\right)=\omega\left(M_{1}\right)=\omega\left(M_{2}\right)=\cdots=M_{0}^{\prime} .
$$

To prove (7.1) we must show that this class (7.3) has only one member, $M_{0}$. Let $\mathscr{X}_{i}$ be the set of all cells occurring in both $\mathfrak{A}_{\Omega}$ and in $M_{i}$, $(i=0,1,2, \cdots)$, that is:

$$
X \in \mathscr{X}_{i} \text { only if } X \in \in \mathfrak{A}_{\Omega} \text { and } X M_{i}=X .
$$

Similarly let $\mathcal{X}_{0}^{\prime}$ be the set of all cells occurring in both $\mathscr{Y}_{\Omega}$ and in $M_{0}^{\prime}$.

Let $\omega\left(\mathcal{X}_{i}\right)$ denote the class of all cells $\omega(X)$, where $X \in \mathcal{X}_{i}$ $(i=0,1,2, \cdots)$. Then it follows from (7.4) together with the definition of $M$ that

$$
\omega\left(\mathscr{X}_{i}\right) \subseteq \mathscr{X}_{0}^{\prime} \quad(i=0,1,2, \cdots),
$$

and hence for the union,

$$
\sum_{i=0,1, \ldots} \omega\left(\mathscr{X}_{i}\right)=\omega\left(\mathscr{X}_{0}\right)+\sum_{j=1,2, \ldots} \omega\left(\mathscr{X}_{j}\right) \subseteq \mathscr{X}_{0}^{\prime} .
$$

Now for each $Y \in \mathcal{X}_{0}^{\prime}$ there exists an $X \in \in \mathfrak{A}_{\Omega}$ such that $\omega(X)=Y$. (This follows from our assumption that $\mathfrak{A}_{\Omega} \subseteq \mathfrak{P}_{\Omega}$.) Such $X$ is evidently an element of one of the $\mathscr{X}_{i}$ (for $i=0,1,2, \cdots$ ), and hence (7.7) may be sharpened to

$$
\omega\left(\mathscr{X}_{0}\right)+\sum_{j=1,2, \ldots} \omega\left(\mathscr{X}_{j}\right)=\mathscr{X}_{0}^{\prime} \text {. }
$$

Now by hypothesis and Theorem $4, \mathfrak{A}_{\Omega}$ satisfies the target condition; therefore, by Theorem 5, Corollary 2,

$$
A B=0 \text { for } A \in \omega\left(\mathcal{X}_{0}\right), \quad B \in \sum_{j=1,2, \ldots} \omega\left(\mathcal{X}_{j}\right) .
$$


Hence $\sum_{j=1,2}, \cdots \omega\left(\mathscr{X}_{i}\right)$ must be an empty class, for otherwise, by (7.8) and (7.9), we should have a disjoint nontrivial separation of the elements of $\mathcal{X X}_{0}^{\prime}$, contrary to Lemma 2. Thus $M_{0}$ is the only member of (7.3) and we have proved (7.1).

To complete the proof that $M \in \mathfrak{B}_{\Omega}$ there remains only to show that for each fixed $\omega \in \Omega$,

$$
\omega(M)=M_{1}
$$

has a solution $M \in M$ for each $M_{1} \in M$. This is however evident from the remarks immediately preceding (7.8), and the proof of Theorem 6 is complete.

Using Theorem 5 on Theorem 6 we have:

THEOREM 7. Let $\mathfrak{A}_{\Omega}^{\prime} \subseteq \mathfrak{A}_{\Omega} \subseteq \mathfrak{P}_{\Omega}$. If $\cap \mathfrak{A}_{\Omega} \in \mathfrak{P}_{\Omega}$ (or equivalently, if $\mathfrak{A}_{\Omega}$ satisfies the target condition), then $\cup \mathfrak{A}_{\Omega}^{\prime} \in \mathfrak{B}_{\Omega}$.

We explicitly note the important special case of Theorem 7:

Theorem 8. A necessary and sufficient condition that the class $\mathfrak{B}_{\Omega}$ of all $\Omega$-permutational partitions of $(U, \Psi: \Omega)$ form a complete lattice, $\left(\mathfrak{B}_{\Omega}, \cap, \cup\right)$, is that $\cap \mathfrak{B}_{\Omega}=\in \mathfrak{P}_{\Omega}$.

As a further special case, since the concepts schlicht and permutational monotation coincide for finite algebras ( $U=$ finite set), Theorems 8 and $3^{\prime}$ immediately yield:

TheOREM 9. The class $\mathfrak{B}_{\Omega}$ of all $\Omega$-permutational partitions of a finite algebra $(U, \Psi: \Omega)$ form a lattice $\left(\mathfrak{B}_{\Omega}, \cap, \cup\right)$.

In this finite case we may further prove:

TheOREM 10. The class $\mathfrak{B}_{\Omega}$ of all $\Omega$-permutational partitions of a finite algebra $(U, \Psi: \Omega)$ is coextensive with the class of all $\Omega$-partitions which have $P=\cap \mathfrak{B}_{\Omega}$ as a refinement.

Proof. Since $\left(\mathfrak{P}_{\Omega}, \cap, \cup\right)$ forms a lattice (Theorem 9 ), we have merely to show that

$$
P \leqq A \text { and } A \in \mathfrak{U}_{\Omega} \rightarrow A \in \mathfrak{P}_{\Omega} \text {. }
$$

Because of the finiteness of the number of cells it is sufficient to prove that: for given $\omega \in \Omega$ and for given $A \in A$, there exists an $A^{\prime} \in A$ such that $\omega\left(A^{\prime}\right)=A$. This is however clearly true; if $P \in P$ and $P \subseteq A$, under our hypothesis there exists a $P^{\prime} \in P$ such that $\omega\left(P^{\prime}\right)=P$. If $A^{\prime}$ is that cell of $A$ defined by $P^{\prime} \subseteq A^{\prime}$, then clearly $\omega\left(A^{\prime}\right)=A$ as required. This proves the theorem.

Note: The theorem says that an $\Omega$-partition which has $P$ as a refinement is necessarily an $\Omega$-permutational partition. It must not however be inferred that a non-permutational $\Omega$-partition of $(U, \Psi: \Omega)$ is necessarily a refinement of $P$. (See $\$ 12$ for a counterexample.) 
For a general algebra, whether or not $\mathfrak{B}_{\Omega}$ is a lattice (see the example preceding Theorem 4$)$, a sub-lattice $(\mathfrak{B}, \cap, \cup)$ of $\left(\mathfrak{U}_{\Psi: \Omega}, \cap, \cup\right)$ in which $\mathfrak{B} \subseteq \mathfrak{P}_{\Omega}$ is simply called a $\Omega$-permutational (sub-) lattice.

If we use the "generative" notation of (2.10) and (2.11), it follows from Theorem 1 that for each given class $\mathfrak{A}_{\Omega}$ of $\Omega$-partitions of the algebra $(U, \Psi: \Omega)$, $\mathfrak{A}_{\Omega} \subseteq \mathfrak{U}_{\Psi: \Omega}:\left(\left|\mathfrak{A}_{\Omega}\right|, \cap, \cup\right)$ is a sub-lattice and $\left(\left\|\mathfrak{A}_{\Omega}\right\|, \cap, \cup\right)$ is a complete sub-lattice of $\left(\mathfrak{U}_{\Psi: \Omega}, \cap, \cup\right)$,

$$
\left|\mathfrak{A}_{\Omega}\right| \subseteq\left\|\mathfrak{A}_{\Omega}\right\| \subseteq \mathfrak{U}_{\Psi: \Omega}
$$

We shall prove the further result:

THEOREM 11. Let $\mathfrak{A}_{\Omega}$ be a class of $\Omega$-permutational partitions, $\mathfrak{A}_{\Omega} \subseteq \mathfrak{B}_{\Omega}$. If glb $\mathfrak{A}_{\Omega}$ is $\Omega$-permutational, $\cap \mathfrak{A}_{\Omega} \in \mathfrak{B}_{\Omega}$ (or equivalently, if $\mathfrak{A}_{\Omega}$ satisfies the target condition), then $\left(\left|\mathfrak{A}_{\Omega}\right|, \cap, \cup\right)$ and $\left(\left\|\mathfrak{A}_{\Omega}\right\|, \cap, \cup\right)$, the simple and complete lattices generated by $\mathfrak{A}_{\Omega}$ respectively, are both $\Omega$-permutational lattices,

$$
\left|\mathfrak{A}_{\Omega}\right| \subseteq\left\|\mathfrak{A}_{\Omega}\right\| \subseteq \mathfrak{P}_{\Omega}
$$

Because of (7.10) the theorem will be proved if (7.11) is established merely for the complete case $\left\|\mathfrak{A}_{\Omega}\right\|$.

Proof. If $\mathfrak{A}_{\mathbb{Q}}=\left\|\mathfrak{A}_{\mathbb{\Omega}}\right\|$, the theorem follows directly from Theorems 5 and 7 . Suppose then that

$$
\mathfrak{A}_{\Omega} \subset\left\|\mathfrak{A}_{\Omega}\right\|
$$

We then have

$$
\mathfrak{A}_{\Omega} \subset \mathfrak{A}_{\Omega}^{*} \subseteq\left\|\mathfrak{A}_{\Omega}\right\|
$$

where

$$
\mathfrak{A}^{*}=\mathfrak{A}_{\Omega}+\left\{\cap \mathfrak{A}_{\Omega}^{\prime}\right\}+\left\{\cup \mathfrak{A}_{\Omega}^{\prime}\right\},
$$

that is, the union of $\mathfrak{A}_{\Omega}$ with the class of glb's and lub's of all subsets $\mathfrak{X}_{\Omega}^{\prime}$ of $\mathfrak{A}_{\Omega}$. By Theorems 5 and 7 ,

$$
\mathfrak{A}_{\Omega}^{*} \subseteq \mathfrak{P}_{\Omega}
$$

Moreover, by the considerations leading to (2.13) together with our hypothesis concerning $\cap \mathfrak{A}_{\Omega}$, we have

$$
\cap \mathfrak{A}_{\Omega}^{*}=\cap \mathfrak{A}_{\Omega}=\in \mathfrak{P}_{\Omega} .
$$

If $\mathfrak{A}_{\Omega}^{*}=\left\|\mathfrak{A}_{\Omega}\right\|$, the theorem follows from (7.15), (7.16), and Theorems 5 and 7 . If $\mathfrak{U}_{\Omega}^{*} \neq\left\|\mathfrak{A}_{\Omega}\right\|$, we have (compare with (7.13))

$$
\mathfrak{A}_{\Omega} \subset \mathfrak{A}_{\Omega}^{*} \subset \mathfrak{A}_{\Omega}^{* *} \subseteq\left\|\mathfrak{A}_{\Omega}\right\|,
$$

where $\mathfrak{A}_{\Omega}^{* *}$ is determined by $\mathfrak{A}_{\Omega}^{*}$ in the same way that $\mathfrak{A}_{\Omega}^{*}$ is determined by $\mathfrak{A}_{\Omega}$ according to (7.14). As before we have 


$$
\mathfrak{A}_{\Omega}^{* *} \subseteq \mathfrak{P}_{\Omega} ; \quad \cap \mathfrak{A}_{\Omega}^{* *}=\cap \mathfrak{A}_{\Omega}=\in \mathfrak{P}_{\Omega} .
$$

Either $\mathfrak{A}^{* *}=\|\mathfrak{A}\|$, in which case the theorem follows as before from (7.18) and Theorems 5 and 7 ; or else $\mathfrak{A}_{\Omega}^{* *} \neq\|\| \mathfrak{R} \|$, in which case the theorem follows by (in general transfinite) inductive repetition of the foregoing argument. This establishes Theorem 11.

8. Closure of $\Omega$. Let $\Omega^{*}$ be the (complete) closure of $\Omega$, that is, $\Omega^{*}$ consists of all monotations of $U$ which may be expressed as the product ( = composition) of a (not necessarily finite) set of $\omega$ 's $\in \Omega$. If we consider $\left(U, \Omega^{*}\right)$ in place of $(U, \Omega)$, a slight reflection shows that

$$
\mathfrak{U}_{\Omega}=\mathfrak{U}_{\Omega^{*}} ; \quad \mathfrak{\Im}_{\Omega}=\mathfrak{\Im}_{\Omega^{*}} ; \quad \mathfrak{B}_{\Omega}=\mathfrak{P}_{\Omega^{*}}
$$

Hence since we are essentially concerned with $\mathfrak{U}, \mathfrak{S}, \mathfrak{B}$, no generality is lost by assuming the system $\Omega$ to be closed, $\Omega=\Omega^{*}$, as we henceforth shall. If $\Omega_{1}$ is a generating subset of such a (closed) $\Omega$, evidently (8.1) insures

$$
\mathfrak{U}_{\Omega_{1}}=\mathfrak{U}_{\Omega} ; \quad \Im_{\Omega_{1}}=\Im_{\Omega} ; \quad \mathfrak{P}_{\Omega_{1}}=\mathfrak{P}_{\Omega}
$$

9. Representations; atoms. If $\Omega$ and $\boldsymbol{\Omega}$ are systems of monotations of a class $U=\{\cdots, x, \cdots\}$ and a class $A=\{\cdots, A, \cdots\}$ respectively, we speak of a strong homomorphism

$$
(U, \Omega) \rightarrow \rightarrow(A, \Omega)
$$

if we have two correspondences

$$
\begin{aligned}
& \omega \rightarrow \boldsymbol{\omega} \\
& x \rightarrow X
\end{aligned} \quad(\omega \in \Omega, \omega \in \Omega, x \in U, X \in A)
$$

for which

$$
\omega \omega^{\prime}(x) \rightarrow \omega \omega(X) .
$$

If both correspondences (9.2) are 1-1, we have a strong isomorphism. A strong homorphism (9.1) in which the $\omega \in \boldsymbol{\Omega}$ are all permutations is called a representation. In a representation, then (because of our closure assumption on $\Omega),(A, \Omega)$ is necessarily a semi-group of permutations of the cells of $\boldsymbol{A}$. If $U$ is finite, for instance, this will be a group, since a finite semi-group of permutations is a group.

Each $A \in \mathfrak{P}_{\Omega}$ furnishes a representation of an algebra $(U, \Psi: \Omega)$ in which the representation set $A=\{\cdots, A, \cdots\}$ consists of the cells of $A$; here $x \rightarrow X$, where $x \in X, X \in A$.

Such a representation assumes an intrinsic significance if $(U, \Psi: \Omega)$ is an atomistic algebra, by which we mean that $\cap \mathfrak{B}_{\Omega}=\in \mathfrak{B}_{\Omega}$. (By Theorem 8 this is equivalent to asserting that $\left(\mathfrak{B}_{\Omega}, \cap, U\right)$ is a complete lattice.) If $(U, \Psi: \Omega)$ is atomistic, we call $P=\cap \Re_{\Omega}$ the atomic partition, the cells of $P$ the atomic cells, and the corresponding representation the atomic representation of 
$(U, \Psi: \Omega)$

We have shown that every atomistic algebra $(U, \Psi: \Omega$ ) (in particular every finite algebra), however involved the (closed) system $\Omega$ of monotations, always represents a semi-group of permutations-in-the-large of $U$, that is, a semi-group of permutations of suitably chosen cells in $U$; every such representation has the atomic representation as a refinement.

10. Invariants. Let $\Pi=\{\cdots, \rho, \cdots\}$ be the group of all permutations of $U$, where $\rho^{-}$is the inverse of $\rho$. In other communications (see $[2,3]$ ) the following notion of transform of an operation was introduced and applied. If $\gamma=\gamma\left(x_{1}, x_{2}, \cdots\right)$ is an operation in the class $U$, the "transform $\gamma^{\prime}$ of the operation $\gamma$ by $\rho$ " is the operation

$$
\gamma^{\prime}=\gamma^{\prime}\left(x_{1}, x_{2}, \cdots\right)=\rho^{-}\left(\gamma\left(\rho\left(x_{1}\right), \rho\left(x_{2}\right), \cdots\right)\right.
$$

which we symbolically write $\rho^{-} \gamma \rho$. For a class $\Gamma=\left\{\gamma_{1}, \gamma_{2}, \cdots\right\}$ of operations

$$
\rho-\Gamma \rho=\Gamma^{\prime}
$$

denotes the class of all transforms of $\gamma_{1}, \gamma_{2}, \ldots$ by $\rho$, and accordingly we speak of the transform $\left(U, \Gamma^{\prime}\right)$ of the algebra $(U, \Gamma)$. Then $(U, \Gamma)$ and $\left(U, \Gamma^{\prime}\right)$ are "equivalent" isomorphic algebras. For instance (see [3]), if $(U, \Gamma)$ is a ring with unit, $(U,+, X)$ and $\rho$ is taken as the permutation $\rho(x)$ $=1-x$, the $\rho$-transform of the ring is $\left(U,+^{\prime}, \times^{\prime}\right)$, where

$$
\begin{aligned}
& x+^{\prime} y=x+y-1, \\
& x \times^{\prime} y=x+y-x y .
\end{aligned}
$$

By an invariant $(\bmod \Pi)$ of an algebra we here understand any property which remains unchanged under the group $I I$ of all "coordinate transformations" in $U$. (See $[2,3]$ for applications.)

We apply this notion to the special case of an atomistic algebra, $(U, \Psi: \Omega)$. With a change $x \rightarrow x^{\prime}=\rho(x)$ of coordinates in $U$ the atomic partition $P=\cap \mathfrak{B}_{\Omega}$ of $U$ will in general differ from the atomic partition $P^{\prime}=\cap \mathfrak{B}_{\mathbf{\Omega}^{\prime}}$ in the new coordinate system. However one may show that the two atomic representations $(\boldsymbol{P}, \boldsymbol{\Omega})$ and $\left(\boldsymbol{P}^{\prime}, \boldsymbol{\Omega}^{\prime}\right)$ are strongly isomorphic, and one has the following theorem.

THEOREM 12. If $(U, \Psi: \Omega)$ is an atomistic algebra, every abstract property (that is, every property which is preserved under strong isomorphism) of the semi-group $(P, \Omega)$ of representing atomic permutations of $(U, \Psi: \Omega)$ is an invariant of the algebra under all coordinate transformations in $U$.

For example the degree (=number of atomic cells, if this is finite) is an invariant. Again, if the semi-group $(\boldsymbol{P}, \boldsymbol{\Omega})$ of atomic permutations is a group (as will always be the case if $U$ is finite), the structure of this group is an invariant of the algebra. Furthermore, if the semi-group $(P, \Omega)$ of atomic permutations is transitive, or intransitive, or primitive, or imprimitive, or 
$r$-fold transitive, and so on, each of these properties is an invariant of the algebra. (Compare with the remarks made in the introduction, concerning the classical case of imprimitivity sets.)

\section{Construction of atoms}

11. Construction of atomic partitions. If $(U, \Omega)$ is atomistic, how can its atomic partition be effectively found? We shall here consider only one special case, where (i) $U$ is a finite set, (ii) $\Omega$ is (compositionally) generable by a single element $\left({ }^{4}\right)$ and (iii) $\Psi$ empty. By analogy with a principal ideal ring we refer to such an algebra as a finite "principal-generator" algebra.

Let then $(U, \Omega)$ be such an algebra, and let $\omega$ be a generator of $\Omega$. Its atomic partition $P$ is given by (see (8.2))

$$
P=\cap \mathfrak{B}_{\Omega}=\cap \mathfrak{P}_{\omega} .
$$

We show how $P$ may be constructed.

For each integer $k \geqq 0$ we define the $k$ th iterative $\omega$-partition $J^{(k)}$ of $U$, by:

$$
x \equiv y\left(J^{(k)}\right) \quad \text { only if } \omega^{(k)}(x)=\omega^{(k)}(y),
$$

where $\omega^{(k)}$ denotes the $k$ th iteration of $\omega$,

$$
\omega^{(k)}(x)=\omega(\omega(\cdots(\omega(x)) \cdots)) \quad \text { (k iterations). }
$$

(Here the 0th iterative partition $J^{(0)}$ is understood to be the null partition 0 (1.6)).

That $J^{(k)}$ as just defined determines an $\omega$-partition is evident since $\equiv$ given by (11.2) is clearly an equivalence relation in $U$, and furthermore

$$
x \equiv y\left(J^{(k)}\right) \rightarrow \omega(x) \equiv \omega(y) \quad\left(J^{(k)}\right),
$$

since patently

$$
\omega^{(k)}(x)=\omega^{(k)}(y) \rightarrow \omega^{(k+1)}(x)=\omega^{(k+1)}(y) .
$$

From (11.4) also follows:

$$
x \equiv y\left(J^{(k)}\right) \rightarrow x \equiv y\left(J^{(k+t)}\right) \quad(t=0,1,2, \cdots),
$$

that is,

$$
J^{(k)} \leqq J^{(k+t)} \quad(t=0,1,2, \cdots) .
$$

Hence in the sequence of all $k$ th iterative $\omega$-partitions of $U$,

$$
J^{(0)}, J^{(1)}, J^{(2)}, \cdots
$$

(4) The case considered in IV may be extended to that in which $\Omega$ has a finite number of generators, $\omega_{1}, \omega_{2}, \cdots, \omega_{f}$. Here the $k$ th iterative partition $J^{(k)}$ must be defined by: $x \equiv y\left(J^{(k)}\right)$ only if $\omega_{i_{1}} \omega_{i_{2}} \cdots \omega_{i_{k}}(x)=\omega_{i_{1}} \omega_{i_{2}} \cdots \omega_{i_{k}}(y)$ for all values of the $i_{t}=1,2, \cdots, f$. With this modification, small changes in the considerations of IV yield a construction of the atomic partition $\boldsymbol{P}$ for such $(U, \Omega)$. 
each partition is a refinement of any succeeding it. However since $U$ is finite, at most a finite number of these divisors can be proper. We have the following theorem.

THEOREM 13. If for some $k, J^{(k)}$ is a permutational $\omega$-partition, then

$$
J^{(k)}=J^{(k+1)} \quad\left(=J^{(k+t)}, t=0,1,2, \cdots\right) .
$$

Conversely, if for given $k, J^{(k)}$ is not a permutational w-partition, then $J^{(k)}$ is a proper refinement of $J^{(k+1)}$,

$$
J^{(k)}<J^{(k+1)}
$$

Proof. Using (11.2), the premise is equivalent to

$$
\omega^{(k)}(x) \neq \omega^{(k)}(y) \rightarrow \omega^{(k+1)}(x) \neq \omega^{(k+1)}(y),
$$

while the desired conclusion is equivalent to

$$
\omega^{(k+1)}(x)=\omega^{(k+1)}(y) \rightarrow \omega^{(k)}(x)=\omega^{(k)}(y) .
$$

If this conclusion were false, that is, if $x, y$ should exist for which the left side of (11.9) held but for which $\omega^{(k)}(x) \neq \omega^{(k)}(y)$, the premise (11.8) would immediately imply the contradiction that the left of (11.9) does not hold. This establishes the direct part of the theorem.

The converse part follows in a similar manner. For, the assumption that $J^{(k)}$ is not $\omega$-permutational is equivalent to: there exist elements $a, b$ in $U$ such that $a \neq \equiv\left(J^{(k)}\right)$ but for which $\omega(a) \equiv \omega(b)\left(J^{(k)}\right)$; that is, such that

$$
\begin{aligned}
\omega^{(k)}(a) & \neq \omega^{(k)}(b), \\
\omega^{(k+1)}(a) & =\omega^{(k+1)}(b) .
\end{aligned}
$$

We show that $a \equiv b\left(J^{(k+1)}\right)$ and hence that $J^{(k)}$ is a proper refinement of $J^{(k+1)}$. For, if $a \neq b\left(J^{(k+1)}\right)$, then $\omega^{(k+1)}(a) \neq \omega^{(k+1)}(b)$, which contradicts our premise. This completes the proof of Theorem 13.

Referring to (11.7) and the accompanying remark, Theorem 13 has the following existence corollary:

Corollary. For some integer $k$, the kth iterative $\omega$-partition $J^{(k)}$ of $(U, \Omega)$ is a permutational partition, $J^{(k)} \in \mathfrak{P}_{\Omega}$.

For otherwise, by the converse part of Theorem 13, (11.7) would represent an infinite sequence of proper refinements

$$
J^{(0)}<J^{(1)}<J^{(2)}<\cdots
$$

in the finite set $U$, which is of course impossible.

The corollary justifies the definition: the characteristic $r\left(=r_{\omega}\right)$ of the monotation $\omega$ is the smallest non-negative integer $J^{(k)}$ for which $J^{(k)}$ is an $\omega$-permutational partition. The permutational $\omega$-partition $J^{(r)}$, also written 
simply

$$
J^{(r)}=J=\{\cdots, J, \cdots\}
$$

we call the characteristic partition of $(U, \Omega)$, and its cells $\cdots, J, \cdots$ the characteristic cells.

We may now restate Theorem 13 in the following form.

THEOREM 14. If $J=$ characteristic partition of $(U, \Omega)$, and if $J^{(k)}=k$ th iterative $\omega$-partition of $U$, then $J^{(k)}$ is $\omega$-permutational, respectively non $\omega$-permutational, according as $J^{(k)}=J$, respectively $J^{(k)}<J$.

12. Relation of iterative to general partitions.

TheOREM 15. Each permutational w-partition $A$ of $(U, \Omega)$ has each iterative partition $J^{(k)}$ as a refinement:

$$
A \in \mathfrak{B}_{\Omega} \rightarrow J^{(k)} \leqq A \quad(\text { for } k=0,1,2, \cdots) .
$$

Proof. It must be shown that

$$
x \equiv y\left(J^{(k)}\right) \rightarrow x \equiv y(A) .
$$

Suppose this false, that is, suppose $a, b$ of $U$ to exist such that

$$
a \equiv b\left(J^{(k)}\right), \quad a \neq \equiv(A) .
$$

Now the first part of (12.2) implies

$$
\omega^{(k)}(a)=\omega^{(k)}(b),
$$

while the second part of (12.2) implies (since $A$ is $\omega$-permutational)

$$
\begin{aligned}
\omega(a) \neq \omega(b)(A), & \rightarrow \omega(\omega(a)) \neq \equiv \omega(\omega(b))(A), \\
& \rightarrow, \cdots, \rightarrow \omega^{(k)}(a) \not \equiv \omega^{(k)}(b)(A) .
\end{aligned}
$$

This is in obvious contradiction with (12.3), and establishes Theorem 15.

ThEOREM 16. The characteristic partition $J$ and the atomic partition $P$ are identical,

$$
J=P=\cap \mathfrak{B}_{\omega}=\cap \mathfrak{P}_{\boldsymbol{Q}} .
$$

Proof. Since $U$ is finite, the atomic partition $P$ exists, and by Theorem 8

$$
P \leqq J
$$

On the other hand, by Theorem 15 we have

$$
J \leqq P .
$$

The theorem then follows from (12.6) and (12.7).

From Theorem 10 we further have that: any $\omega$-partition of $U$ which has 
$J(=P)$ as a refinement is necessarily a permutational $\omega$-partition. On the other hand, the non-permutational $\omega$-partitions $J^{(0)}, J^{(1)}, \cdots, J^{(k)}$ (for $k<r=$ characteristic of $\omega$ ) were seen to be refinements of $\boldsymbol{P}$. However, as remarked in $\$ 7$, following Theorem 10 , not all non-permutational $\omega$-partitions of $U$ need be refinements of $P$. For instance, if $U=\{1,2,3, \cdots, 8\}$ and if $\Omega$ is generated by $\omega$ taken as

$\frac{x}{\omega(x)} \mid$\begin{tabular}{llllllll}
1 & 2 & 3 & 4 & 5 & 6 & 7 & 8 \\
\hline 1 & 2 & 2 & 2 & 6 & 5 & 3 & 7
\end{tabular},

one finds the characteristic (=atomic) cells of $U$ to be

$$
P ; \quad P_{1}=\{1,2\}, \quad P_{2}=\{3,4,5,6,7,8\},
$$

and the characteristic permutation $\omega$ to be the identity $\omega=\left(P_{1}\right)\left(P_{2}\right)=1$. One easily verifies that $B=\left\{B_{1}, B_{2}, B_{3}\right\}$, with

$$
B_{1}=\{1\}, \quad B_{2}=\{2,3,4,7,8\}, \quad B_{3}=\{5,6\},
$$

is a non-permutational $\omega$-partition of $U$, in which evidently $B \nless \mathfrak{B}$.

In the foregoing construction for $P$, explicit use was made of the generator $\omega$ of $\Omega$. If another generator $\omega^{\prime}$ (exists and) is chosen, the various corresponding iterational partitions $J^{\prime(k)}$ will in general differ from the $J^{(k)}$. However the characteristic and the characteristic partition must be the same in each case,

$$
r_{\omega}=r_{\omega^{\prime}} ; \quad J=J^{(r)}=J^{\prime}=J^{\prime\left(r^{\prime}\right)}=P,
$$

since the atomic $\Omega$-partition is unique. One may thus speak of the characteristic $r$ of $\Omega$, and this is an invariant of the algebra $\left({ }^{4}\right)$.

We conclude with a simple illustration. Let

$$
U=\{0,1,2, \cdots, 12\}
$$

and let $\Omega$ be generated by

$$
\omega(x)=x^{2}(\bmod 13)
$$

Here

$$
\begin{aligned}
& J^{(1)}=\left\{J_{0}^{(1)}, J_{1}^{(1)}, \cdots, J_{6}^{(1)}\right\}, \text { with } J_{0}^{(1)}=\{0\}, J_{i}^{(1)}=\{i, 13-i\},(i=1,2, \cdots, 6), \\
& J^{(2)}=\left\{J_{0}^{(2)}, J_{1}^{(2)}, \cdots, J_{3}^{(2)}\right\}, \text { with } J_{0}^{(2)}=\{0\}, J_{1}^{(2)}=\{1,5,8,12\}, \\
& J_{2}^{(2)}=\{2,3,10,11\}, J_{3}^{(2)}=\{4,6,7,9\} .
\end{aligned}
$$

One finds that $J^{(3)}=J^{(2)}$, and hence the algebra $(U, \Omega)$ is of characteristic 2 , and the atomic partition is $P=J\left({ }^{2}\right)=J$. The characteristic permutation $\omega$ is seen to be: $\omega=\left(P_{0}\right)\left(P_{1}\right)\left(P_{2} P_{3}\right)$. 


\section{BibLIOGRAPHY}

1. Garrett Birkhoff, Lattice theory, Amer. Math. Soc. Colloquium Publications, vol. 25.

2. Alfred L. Foster, Maximal idempotent sets in a ring with unit, Duke Math. J. vol. 13 (1946) pp. 247-258.

3. - The theory of Boolean-like rings, Trans. Amer. Math. Soc. vol. 59 (1946) pp. 166187. Additional references are listed in this paper.

University of California, Berkeley, Calif. 